\title{
Critical features about urban pedestrian crossings: a case study
}

\author{
M. Lo Presti, B. Lo Casto \& F. Montano \\ Department of Energy (Transportation Engineering), \\ Palermo University, Italy
}

\begin{abstract}
The theme of safety for vulnerable road users (disabled, elderly, and children) in urban areas plays a role of primary interest for many implications in quality of life. The deficiency of safety on city streets is primarily due to the simultaneous presence of different components of traffic in an increasingly complex and inappropriate ambient for their cohabitation. In urban areas the road intersections, which are crossed by different categories of traffic (motorized vehicles, pedestrians, non-motorized vehicles), represent a critical node, both in terms of quality of circulation that of road safety. In recent years there has been the $76.62 \%$ of accidents in the urban area, with the $13 \%$ of accidents involving pedestrians of which about $30 \%$ of accidents occurred near intersections. All these reasons explain the complexity in the planning of an intersection, particularly when vulnerable users are involved. In this paper we propose an applicative study of "pedestrian crossings", regulated by traffic lights or free, and what factors affect their planning and design to achieve an optimal quality of service and high safety standards. The study area involves one of the main streets of city of Palermo, which link the city center and some of the suburbs, focusing on the design of pedestrian crossings and their location in the urban context, starting by existing legislation in order to achieve an optimization of the number of pedestrian crossings, the distance between them and traffic light cycle if present. In particular we can design a possible new layout of pedestrian crossings, starting by analysis the current configuration and its critical points and simulating different future scenarios that take into account the dual role of the street inside and outside the district and in the whole urban network.
\end{abstract}

Keywords: safety, pedestrian crossing, critical features. 


\section{Introduction}

The theme of the safety of vulnerable road users in urban areas plays a role of primary interest because of the many implications it has on quality of life.

The analysis data provided annually by ISTAT (Istituto Nazionale di Statistica National Institute of Statistic) shows that Italian cities are the physical place where the highest number of accidents occur.

ISTAT has recorded the last two years in an urban area 176,897 accidents per year $(76.62 \%$ of total), of these about $13 \%$ involves pedestrians with 264 deaths (equal to $10: 57 \%$ of total deaths) and 9,765 injured (equal to $40.90 \%$ of total injuries).

The deficiency safety on urban streets is primarily due to the simultaneous presence of different components of traffic in an ever more complex and inappropriate ambient for their cohabitation.

This paper proposes a study of the "pedestrian crossings", regulated by traffic light or free, and what factors affect their proper planning and design to achieve an optimal quality of service and high safety standards.

\section{Vulnerable road users}

Vulnerable road users are identified in a group of people with specific characteristics and specific behavior patterns, linked by the common difficulty of moving in an environment not always designed for them.

They are recognized in several categories related to their motor skills and different abilities that determine their level of vulnerability. The most vulnerable are the disabled, elderly and children.

The disabled and the elderly, whose behavior is closely related to their health that reduces motor skills and reaction in critical situations, are the categories that in the event of an accident suffer more damage because of the slow recovery capabilities. Children under ten years (Sandels 1976) are more exposed and less visible because their behavior sometimes impulsive and unwise.

\section{Road intersections and pedestrian crossings}

By definition a road intersection is an infrastructural configuration in which two or more roads intersect with the subsequent partial or total exchange of traffic flows. The intersections in urban area are concerned by different categories of traffic (motor vehicles, pedestrians, non-motorized vehicles, etc...) that contribute to make the intersection a critical point, both for the quality of traffic and for the road safety.

Statistical data, issued by ISTAT, show that about $30 \%$ of road accidents occur in urban areas near intersections.

This suggests the complexity that resides in the design process of an intersection, particularly when it involves vulnerable road users. 


\section{Reference rules}

The Italian Road Laws are included in D. Lgs. 285/1992 and D.P.R. 495/1992 and ss.mm.ii. We report an extract of these rules about pedestrian crossing and their safety:

\section{Art.3 : Pedestrian crossing}

[...] part of street, opportunely indicated, in which pedestrians can walk from one side to other side of the street and have right of way respect to vehicles.

Art. 145

[...]Pedestrian crossings are indicated through with zebra lines, they have length $2.50 \mathrm{~m}$, in local streets, and $4.00 \mathrm{~m}$ in other streets; lines are $50 \mathrm{~cm}$ large.

[...] Length of pedestrian crossing must be however proportional to number of pedestrian.

[...] vehicles must stop and give right to way to pedestrian on pedestrian crossing; stop line, if it exists, must be $5.00 \mathrm{~m}$ before pedestrian crossing

[...] in the streets where parking is authorized, for having a better visibility, a yellow zig zag line could be ahead to pedestrian crossing. On this line parking is forbidden

\section{Art. 190: Pedestrian behaviour}

[...] pedestrian must use pedestrian crossing ... when they exist ...pedestrian can cross the street only in perpendicular direction with the necessary attention to avoid dangerous for them or for others.

[...] It's forbidden to pedestrian cross the streets in diagonal way

[...] Pedestrian that are crossing without pedestrian crossing must give right to way to vehicles.

\section{Art. 141: Behaviour to pedestrian}

[...] The driver must reduce the speed and stop the vehicle near pedestrian crossing and however when pedestrians are uncertain

\section{Art. 153: Reflecting elements as integration of horizontal signals}

[...] Reflecting elements can be used to enforce horizontal signals.

[...] They must have the body and the reflecting part of the same color of the horizontal signal.

[...] Reflecting elements cannot be taller than $2.5 \mathrm{~cm}$ and must be fixed to the street with systems that do not allow their detachment by the traffic.

\section{Art. 191}

[...] When traffic is not regulated by policeman or traffic lights, drivers must stop when pedestrian are crossing the street on pedestrian crossing. Besides they must give right to way, slowing down or stopping, to pedestrian that are crossing or that get ready to cross the street.

[...] If there are not pedestrian crossing and if pedestrian are crossing the street, drivers must leave them to reach the other side of the street in safety conditions. [...] Pedestrian that are crossing a street where there are not pedestrian crossing, must give right to way to drivers. 


\section{Analysis of pedestrians and motorists behaviour}

The pedestrian feels himself psychologically inferior in relation with all other road users because of his greater vulnerability in the "road". In fact more often "he doesn't consider himself a road user for his vulnerable nature" (Pasanen 1992), thus contributing to an increase in his sense of fear and discomfort when moving in an environment that does not feels safe.

The pedestrian achieves a process of choice, finalized to minimize the generalized cost of his move, when crossing a road:

- the choice of the node on which to make the crossing;

- choice of route to reach the point of crossing;

- identify the timing for the crossing.

For the pedestrian to be decisive in this phase the distance of the node from his position, because he is the supplier of motive power for movement, and physical characteristics of route to achieve it (more or less pleasant path, comfort level, perceived level of security, etc.)

The process of choice is influenced by a number of factors:

- type of user who makes the move;

- $\quad$ origin and destination of travel;

- reason for trip (work, study, leisure, etc.);

- urban environment where the moving occurs (area attractiveness);

- physical environment (type of road, roadway width, presence of pedestrian island, etc.);

- presence of children in tow;

- involvement in road accidents in the past;

- number of pedestrians waiting to cross.

The pedestrian crossings will ensure:

- recognition by all users;

- accessibility for all users;

- connecting of strategic points in order to ensure efficiency and functionality of the network.

The car driver generally "does not show any willingness to give priority to the pedestrian in crosswalks" (Varhelyi 1998).

The car driver near pedestrian crossings with the presence of pedestrians makes a choice that is expressed in the following behaviors:

- maintain the same speed;

- reduce the speed;

- accelerate;

- stop.

The process of choice is influenced by:

- $\quad$ speed-distance (Hamed 2001); 
- perceived distance from the edge of the pedestrian sidewalk;

- presence of pedestrian on zebra crossings;

- the pedestrian has not seen the car coming;

- vehicle size;

- physical environment (type of road, roadway width, presence of pedestrian island, etc.);

- frequency of zebra crossings;

- presence of children;

- involvement in road accidents in the past;

- number of pedestrians waiting to cross.

The car driver as opposed to the pedestrian, feeling less vulnerable, feels he has control of the road, not respecting in most cases the most vulnerable users. Generally he tends to reduce his speed or stop when:

- his speed is low;

- he perceives that the pedestrian has not seen him;

- there is a group of pedestrians and not only one pedestrian;

- the pedestrian has already started to cross;

- the pedestrian makes a stop sign with his hand.

Instead, he tends to keep the same speed or accelerate when:

- his speed is supported;

- there is only one pedestrian;

- the pedestrian has not started to cross.

Paradoxically, the driver uses his traveling speed to send messages to other road users; in fact, by accelerating he warns the pedestrian that he will not stop.

\section{A case study: the design of pedestrian crossings}

The study area is a district of Palermo city. Palermo is a city of $158 \mathrm{~km}^{2}$ and a population of about 1.220.000 inhabitants, but 700.000 in an urban area (219.972 households) with a large historical center (about $2,7 \mathrm{~km}^{2}$ ).

\subsection{Definition of the study area and sample}

The area selected for this study is one of the main streets of a residential neighborhood of Palermo: the "Viale Strasburgo".

The avenue, which stretches for about $2.5 \mathrm{~km}$, represents the main axis of the neighborhood, as well as serving as an axis of penetration and connection between the center of the city and some suburbs. 


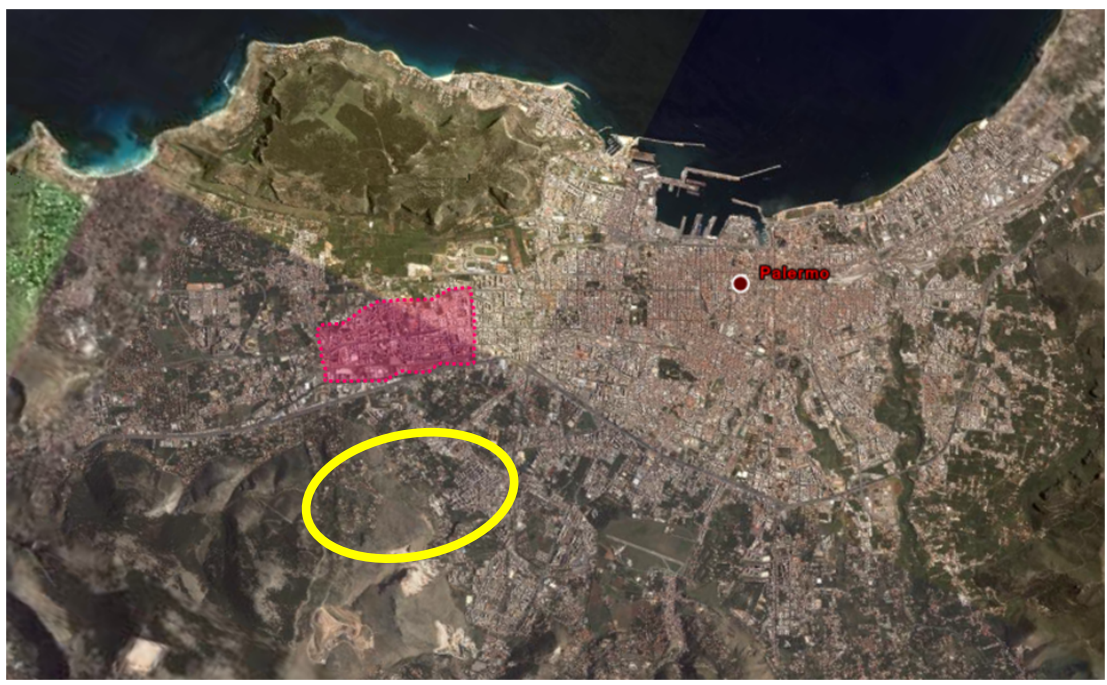

Figure 1: $\quad$ Study area.

The neighborhood around the street (area of $9.69 \mathrm{~km}^{2}$ ) was an area of marshland. It has now been replaced by large modern buildings, and is one of the neighborhoods with the highest number of residents. The district has developed enormously in the last decades, especially in terms of offering services for citizens (primary and secondary schools, municipal delegations, banks, post offices, clinical ambulatory, etc.) and commercial activities (shops, supermarkets, cinemas, gymnasiums, etc.). In particular, the lower end of Viale Strasburgo, up to the intersection with the "Viale Francia", is one of the richest commercial areas of the city.

The district, due to the presence of services and activities, is a "city within a city", and the Viale Strasburgo, as well as being the physical space around which the district lives, performs the function of a penetration route in the urban tissue for the outlying suburbs. In the urban structure of the city the street is probably the only urban axis that can play this role, both for its geographical location within the city of Palermo and for its size compared to other axes. This dual function involves the simultaneous presence of great flows of traffic (which exceed the capacity of the road even outside the peak hour) and pedestrian and non-motorized flows, with the consequent generation of a number of criticalities which impact on life quality of the population (noxious emissions, safety, etc.) and quality of circulation. In addition, the street crosses the "Via Belgio", which is an important access point for flows coming from outside and an exit point for flows from the city.

In particular, we chose a section of the street, from "Viale Francia" to "Via Belgio", where the activities that generate pedestrian flows are more concentrated (schools, shops, banks, etc.). 


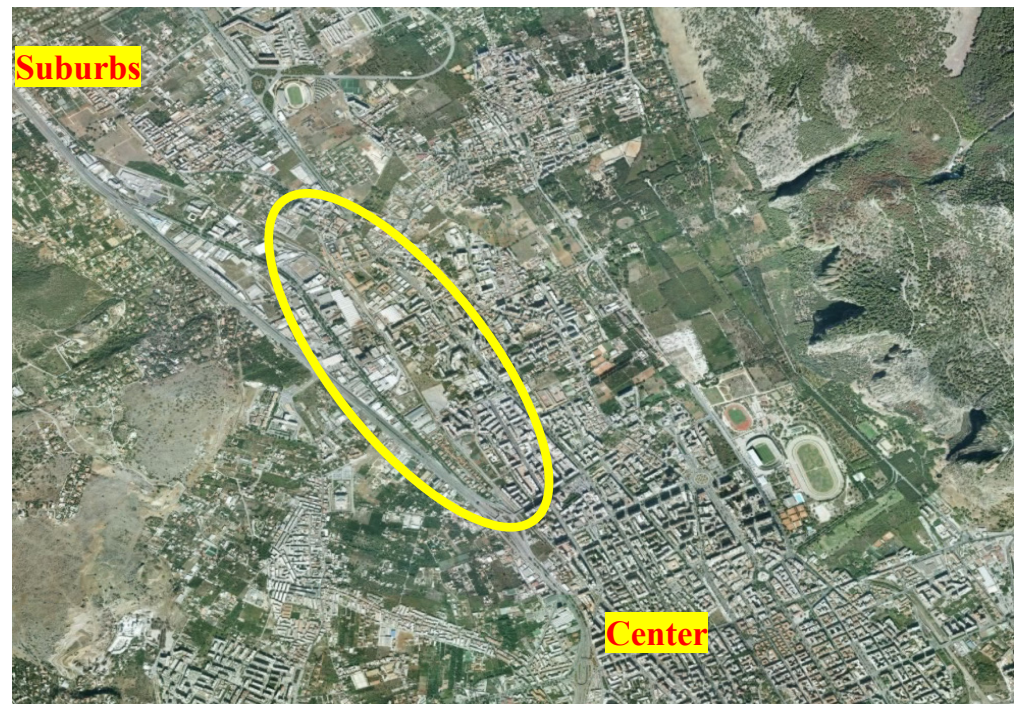

Figure 2: Axis of penetration.

In the section, along approximately $666 \mathrm{~m}$, there are (see fig. 3 in which B: bus stop; red arrow: observation point):

- n. 3 intersections with traffic light;

- n. 5 pedestrian crossings with traffic lights;

- n. 8 pedestrian crossings without traffic lights;

- $\quad$ n. 6 stops of public transport (3 per direction).

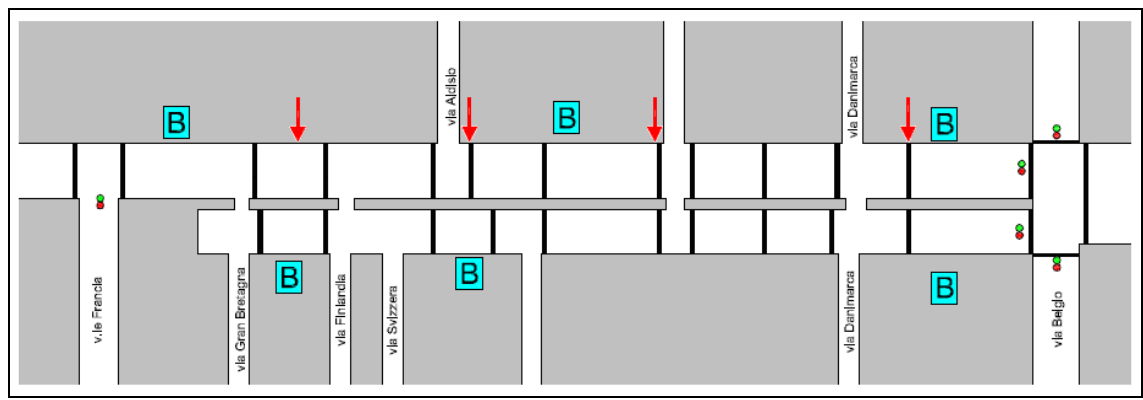

Figure 3: $\quad$ Survey area.

The section under study shows several architectural barriers for the pedestrian (see fig. 4). There is a total absence of spaces for waiting for the green signal, the zebra crossings are small and not very visible to their state, the walkways are full of obstacles of all types (publicity poles, bins, low walls, light poles and traffic lights, flower beds, shops, parked cars, etc.). In addition, other critical points are encountered: the pedestrian crossings are too many (sometimes mutual distances 
are less than $20 \mathrm{~m}$ ) and badly localized in relation to public transport stops, thus helping to create a state of stress for the car driver who is forced to make many stops during his trip. All this contributes to ensuring no minimum standards of safety for vulnerable road users.
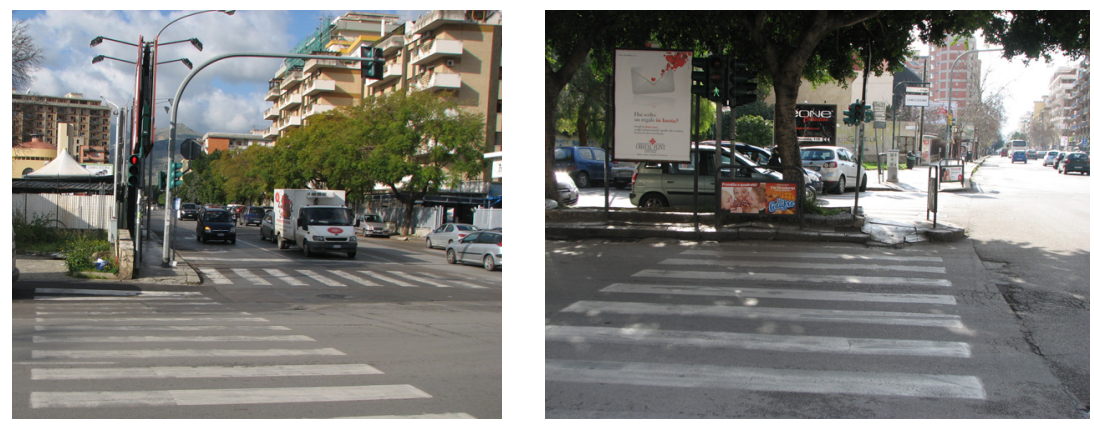

Figure 4: Pedestrian crossings in the study area.

The sample is composed of a heterogeneous group of people who move during the day for various reasons. At peak times the greater flows are composed of students for the presence of two major secondary schools, while in the afternoon, shops, cinema and fitness center attract a large number of pedestrians for fun.

\subsection{Data collection}

The surveys for data collection were undertaken in two different periods during the month of November 2010 and the month of January 2011.

For the type of activities that take place during the day three different time periods were chosen:

1. $8: 00-9: 00$

2. $12: 30-13: 30$

3. 18:00-19:00

The observations were made on 5 zebra crossings for a time of $15 \mathrm{~min}$. in each point of observation (see fig. 5 and table 1).

Table 1: Data collection.

\begin{tabular}{|c|c|c|c|c|}
\hline Period & Pedestrians & Cars & Bus \\
\hline $8: 00-9: 00$ & 752 & 247 & 18 \\
\hline $12: 30-13: 30$ & 814 & 289 & 12 \\
\hline $18: 00-19: 00$ & 374 & 358 & 10 \\
\hline
\end{tabular}




\subsection{Analysis of results}

By data analysis it is visible that just a little more than $60 \%$ of pedestrians make a legal crossing using crossing lines and respect the traffic light signal, while more than $35 \%$ of pedestrian make an illegal crossing outside crossing lines and without the green signal.

Analysis of the data shows that:

Table 2: $\quad$ Pedestrian behaviour.

\begin{tabular}{|c|c|}
\hline Pedestrian crossing with traffic lights & $\mathbf{\%}$ \\
\hline Respect for the green signal & 83 \\
\hline No respect for the green signal & 17 \\
\hline Pedestrian crossing without traffic lights & $\mathbf{\%}$ \\
\hline Crossing in the zebra crossing & 62 \\
\hline Crossing outside the zebra crossing & 38 \\
\hline
\end{tabular}

Instead, drivers have a less legal behavior than pedestrians. In fact, data shows that just $27 \%$ of these slow down or reduce the speed giving right of way to pedestrians. These results are so far from minimal standards that could guarantee pedestrian safety.

Table 3: $\quad$ Car driver behaviour.

\begin{tabular}{|c|c|}
\hline Car driver behavior & $\%$ \\
\hline Speed reduction & 11 \\
\hline Braking & 16 \\
\hline Same speed & 57 \\
\hline Acceleration & 16 \\
\hline
\end{tabular}

\section{Project proposals}

Project proposals are focused on pedestrian safety through a relocation and modification of pedestrian crossings in the study area.

Our proposal is to increase dimensions of pedestrian crossings from $2.50 \mathrm{~m}$ to $4.50 \mathrm{~m}$, to modify the actual location of these using a more rational positioning through all the length of the road.

Besides that, we propose inserting a stop line 5 meters before the pedestrian crossing with reflecting elements for having a dual function of visual and audio signal for drivers. It is possible to see these proposals in figures 5 and 6 .

In conclusion, we can say that these proposals aim to reach a higher level of safety perceived by pedestrians on the road we studied; this is reached through a new design and position of crossing lines and constructive features.

Besides that, we consider that, if crossing lines were put at a different distance from each other, the vehicular traffic will have many benefits. 


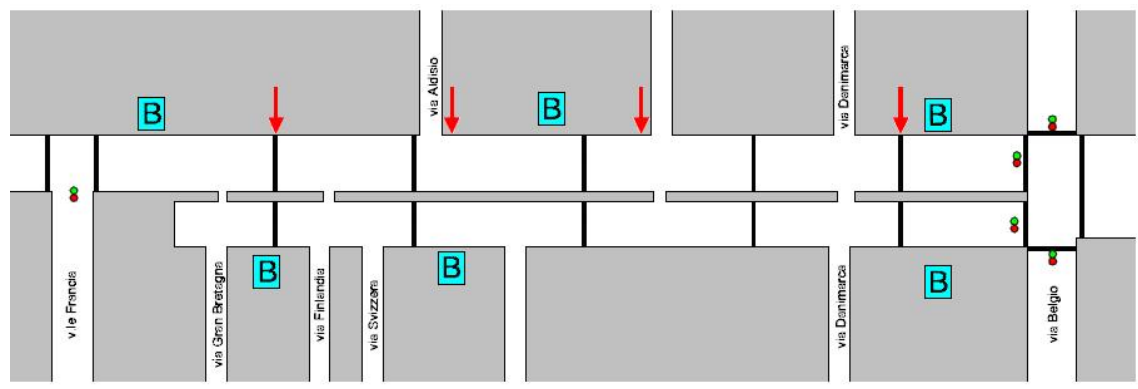

Figure 5: $\quad$ Project proposals.

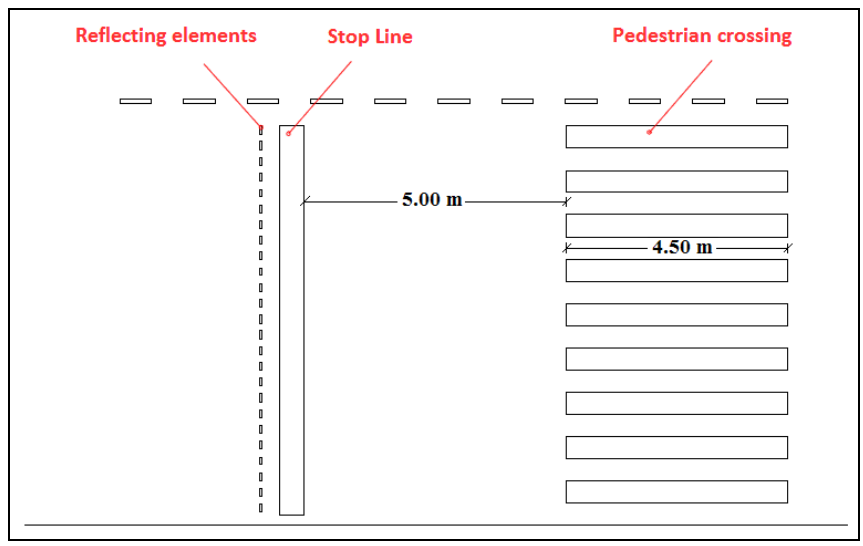

Figure 6: Particular of proposed configuration.

\section{References}

[1] Busi, R.; Zavanella, L., (2001), "La protezione del pedone negli attraversamenti stradali”- Vol.I, Egaf - Forlì

[2] Busi, R.; Tira, M., (2001), "Safety for pedestrians and two wheelers"Quaderno n. 3, Cescam - Brescia

[3] Busi, R.; Maternini, G., (2004), "Le normative sulla progettazione stradale e l'analisi di sicurezza”- Vol.VI, Egaf - Forlì

[4] Certu (1982), "Securité des piétons lors de leur traverse de chausseés"dossier n.21 - Paris

[5] Certu (1988), “Chausseés piétonnes: conception des structures”- Paris

[6] Certu (1990), "Vivre et circuler en ville"'- Paris

[7] Cascetta, E., (1998), "Teoria e metodi dell'Ingegneria dei Sistemi di Trasporto”, UTET - Torino

[8] Deng, W.; Yang, J.; Wang, J.; Li, Q.; Wang, Z., (2006), “Modeling pedestrians' road crossing behavior in traffic system micro-simulation in China", Transportation Research part.A, vol. 40, issue 3 - Elsevier 
[9] Hamed Mohammed, M., (2001), “Analysis of pedestrians' behavior at pedestrian crossing”, Safety Science, vol. 38, issue 1 - Elsevier

[10] Hine, J., (1996), "Pedestrian travel experiences: Assessing the impact of traffic on behaviour and perceptions of safety using an in-depth interview technique”, Journal of Transport Geography, vol. 4, issue 3 - Elsevier

[11] Ignaccolo, M.; Caprì, S., (2000), "Accessibilità e mobilità pedonale nell'isola di Ortigia", Atti della VII conferenza "Vivere e camminare in città", Università degli Studi di Brescia

[12] King J., Soole, D.; Ghafourian, A., (2009), "Illegal pedestrian crossing at signalised intersections: Incidence and relative risk", Accident analysis and prevention, vol. 41, issue 3 - Elsevier

[13] Maternini, G., (1994), "la sicurezza del pedone in città. Il caso di Brescia", Sintesi - Brescia

[14] Maternini, G., (1994), "la sicurezza del pedone in città. Il caso di Brescia", Sintesi - Brescia

[15] Maternini, G., (2000), “Indicatori di sicurezza delle strade urbane”, rivista Le Strade, n.11, La Fiaccola

[16] Maternini G., Rabino G., (2000), "Pianificazione e modellistica della mobilità pedonale", Atti della VII conferenza "Vivere e camminare in città", Università degli Studi di Brescia

[17] Nasar, J., (2003), "Prompting drivers to stop for crossing pedestrians", Transportation Research part. F, vol. 6, issue 3 - Elsevier

[18] Oxley, J.; Ihsen, E.; Fildes, B.; Charlton, J.; Day, R., (2005), “Crossing roads safely: An experimental study of age differences in gap selection by pedestrians", Accident analysis and prevention, vol. 37, issue 5 - Elsevier 